\title{
Coordinated Voltage-Reactive Power Control Schemes Based on PMU Measurement at Automated Substations
}

\author{
Yun-Hyuk Choi*, Sang-Gyun Kang** and Byongjun Lee ${ }^{\dagger}$
}

\begin{abstract}
This paper deals with methodology to control an automatic substation system. The control system can predict the power system condition by a voltage stability index (VSI). The strategies in this paper is called as Voltage-Reactive Power Control (VRPC), which regulates an abnormal voltage of a target substation by using coordination between tap changers and shunt capacitor/reactor. This method is efficient for better voltage profile. The monitoring substation includes whole of substations around the contingency event. The control quantities of the monitoring substations are decided by the calculation of the VSI, called as a Z-index. Case studies with BC Hydro-Quebec system are presented to illustrate this approach using real-time simulator.
\end{abstract}

Keywords: VRPC, PMU, VSI, V-Q plane, Coordinated control

\section{Introduction}

Nowadays the power system varies every time and operates close to its limit. This is mostly attribute to request better power quality at customer side, increase of the power system losses and control utilities installation constraint. In order to maintain power quality and to minimize transmission losses, grid operator controls voltage and reactive power. Although this voltage and reactive power control problem has been important in power system operation, it has become difficult to handle voltage and reactive power because system more and more complex. From this reason, the consequence is that efficient operate system is needed [1-3].

A Substation Automation System (SAS) is suitable for stable operation of power system. It includes real-time monitoring and control, network visualization and warning alarms, distribution system maintenance, and etc. The SAS is composed mainly of three part, which are monitoring, operation, and control. The main function of the SAS is as shown in Fig. 1. Its important factors of monitoring are power element, devices operation, switching device, system fault detect, and relay operation. Breaker control, voltage control, fault remove, and system recovery are operation part of the SAS. Control devices are bus breaker, under-load tap changers (ULTC), switched shunt capacitor (S.C), and switched shunt reactor (Sh.R). Especially the framework of the SAS in view of voltage and reactive power control are high operating flexibility for accurate and rapid monitoring the load condition. And it is

$\dagger$ Corresponding Author: Department of Electrical Engineering, Korea University, Korea. (leeb@korea.ac.kr)

* Department of Electrical Engineering, Korea University, Korea. (lover9000p@korea.ac.kr)

** School of Electrical and Railway Engineering, Kyungil University, Korea. (sgkang@kiu.ac.kr)

Received: November 29, 2014; Accepted: February 5, 2015 improving the system efficiency and the power quality at customer side.

The purpose of voltage control in distribution network is to compensate for load variations and events in the transmission system, such that all customer supply voltages are kept within certain bounds. A number of ULTC, each capable of regulating the voltage of the secondary side of a transformer at one point in $\mathrm{v}$ network, are available in the distribution systems for this purpose. The substation is supplied through cascaded tap changing transformer. But most of the tap operations made in the substation counteract the effect of tap changers higher up in the network. Poor coordination of cascaded tap changers can be dangerous from the voltage stability point of view [4-6].

A new on-load tap changers (OLTC) voltage control strategies are considered for use on networks where distributed generator is connected. Voltage control of distribution system is achieved by using OLTC and S.C. The OLTC and S.C keep the voltage at the substation bus constant [7-9].

The voltage problem is a predominating local problem, so is requested an automation and coordination of reactive power resources at substation. For coordinated voltagereactive power controls at automated substations, real-

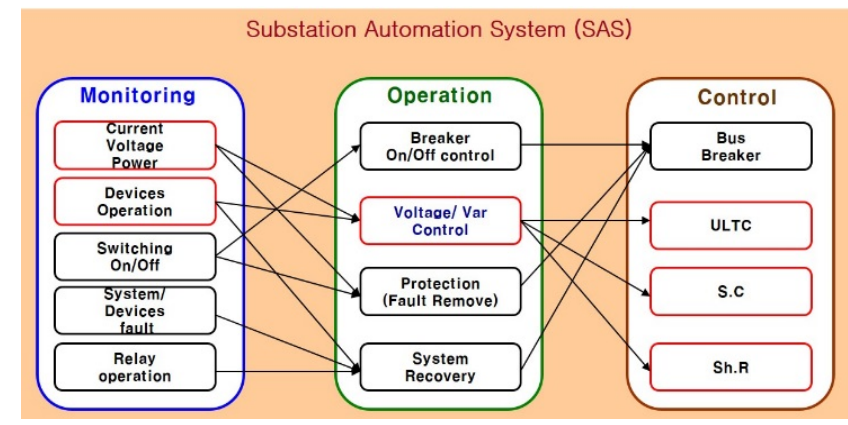

Fig. 1. The main function of substation automation system 
time monitoring (voltage, current, and instability index) is essential. Also, devices modeling for voltage-reactive power control have to be well made [10-12].

This paper proposes the efficient control strategy of ULTC, S.C and Sh.R at a local automated substation. The control methodology is divided into two parts. First part is that system operator judges system conditions using the VSI which is stable or unstable. And if the index exceeds a criterion, which is an abnormal condition, another strategy is needed. So, required reactive power or voltage magnitude are calculated by an index margin and a corresponding tap is blocked. Second part is a thing how to control ULTC, S.C and Sh.R. If a system disturbance occurs, an operation point of post contingency will shift into dead-band of control plane for keeping up a reference value of voltage and reactive power. This control's objective is restoring the subsystem condition to pre-disturbance state.

\section{Automated Substation Control}

On-line substation control is difficult because operators must estimate system condition, stable or unstable, using real-time data. This technique is called by Phase Measurement Unit (PMU) technique [13]. An integrated voltage and reactive power control at automated substations is described as Fig. 2.

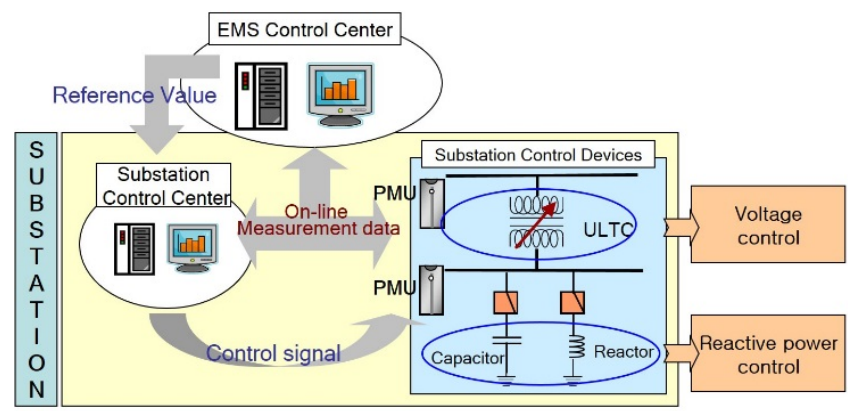

Fig. 2. Automated Substation Control

EMS control center obtains an on-line measurement data from PMU installed in substation and then sends the reference voltage to substation control center. When receiving the value, it also sends the control signal to the substation. Tap changers operates for voltage control and shunt devices operates for reactive power control.

\section{Variable Time Delay Method}

\subsection{Time integration method}

ULTC and S.C are slowly acting. Discrete devices change the tap by one step at a time. If the voltage error remains outside a dead-band longer than a specified delay, ULTC

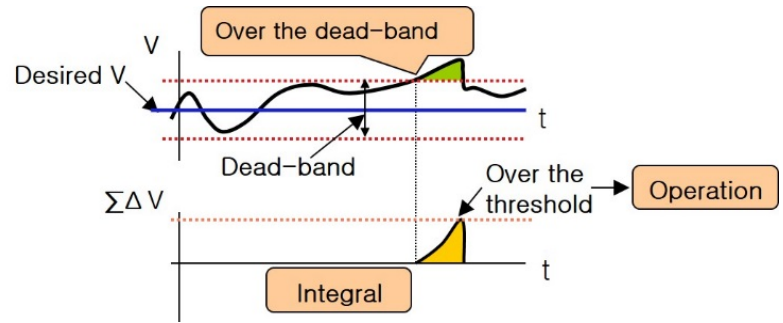

Fig. 3. The concept of time integration method

and S.C are operated. A time delay is composed of a mechanical time delay and an intentional time delay. Ordinarily, the mechanical time delay is fixed. However the intentional time delay, which is to avoid unnecessary tap changes, is different from the mechanical time delay and can be either fixed or adaptive. The time delay becomes shorter for larger voltage errors. General equation about time delay is as follows.

$$
\Delta T_{0}=T_{d 0} \frac{d}{\left|V_{2}-V_{2}^{0}\right|}+T_{f 0}+T_{m}
$$

First term means a variable time delay, second term is a fixed time delay, and third term includes the mechanical time delay, respectively. When the voltage difference is large, the variable time delay enables the voltage to recovery rapidly according to short time delay. This indicates an inverse-time characteristics. The first time delay is a fixed value, and then the next time delay will be reflected from the inverse-time characteristics.

Because of considering the voltage difference, the tap change signal generates for an instantaneous voltage deviation. The fixed time delay dose not actively react owing to operating at a given time. In order to solve this problem, the time integration of the voltage magnitude is used. The concept of the basis algorithm is as shown in Fig. 3.

If the voltage over the dead-band, integration will be started. When the integrated value over the threshold, the device is operated. Using the time integrated value, the controller can handle the intentional delay time depending on the approaching time to threshold. The voltage from load-side and the reactive power from network side are integrated, and S.C/Sh.R or ULTC are selected by operating signal.

\subsection{ULTC and S.C/Sh.R control schemes}

In order to apply the concept of the time integration method that is mentioned in previous section, ULTC is integral to the voltage change of the secondary side and S.C/Sh.R is integrating the reactive power variations of the primary side.

The concept of ULTC control is as shown in Fig. 4. ULTC maintains the load-side which is V2. If the V2 is 

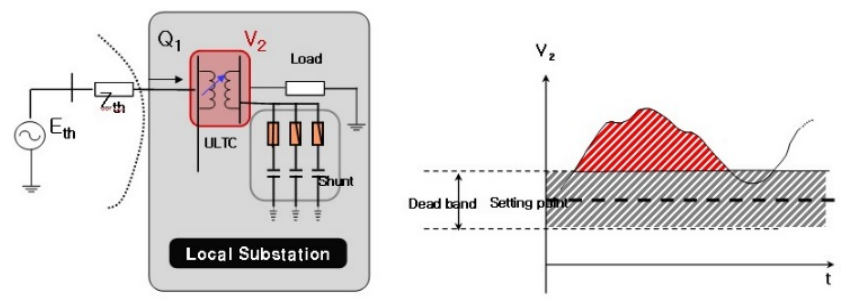

Fig. 4. The concept of ULTC control
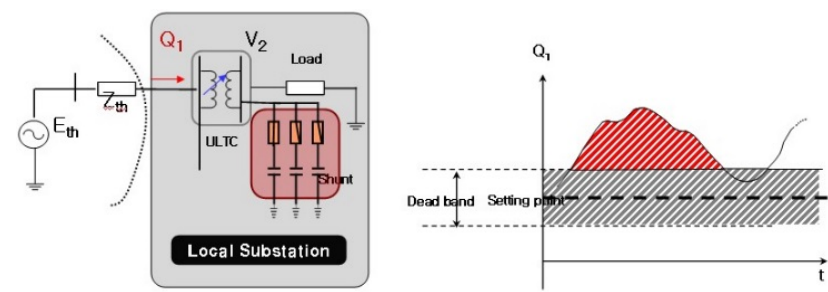

Fig. 5. The concept of S.C control

over the dead-band, the value is integrated. If the integral value is over the threshold, the control signal is generated. The dead-band and threshold are determined by the system operator.

The concept of S.C control is illustrated in Fig. 5. S.C maintains grid side which is Q1. If the Q1 is over the deadband, the value is integrated. If the integral value is over the threshold, the control signal is generated. The deadband and threshold are determined by the system operator.

\subsection{Variable time delay method}

ULTC and S.C/Sh.R control using the time integration method shows fast tap changing with respect to the voltage change relatively. However, if the bus voltage does not deviate significantly in dead-band, it is insensitive to tap changing than fixed time delay. From this reason, variable time delay method, which is a combination of the time integration method and fixed time delay, is used in this paper.

When the integral value of the bus voltage within the outside of the dead-band is greater than the threshold, the control signal is generated using time integration method. On the other hands, if the time duration of the bus voltage within the outside of the dead-band is greater than the threshold, the control signal is generated using fixed time delay control.

Tap oscillation of cascading ULTC in radial networks can be avoided using revised tuning. It is gradually longer delay times for lower level substation. An adaptive time characteristics which combine constant time delay with time integration method is used. Time integration method is useful for large voltage errors. This method will adversely affect for smaller voltage errors that is close to dead-band. In this case, constant time delay becomes shorter than time integration method. This formulation is described as Fig. 6.

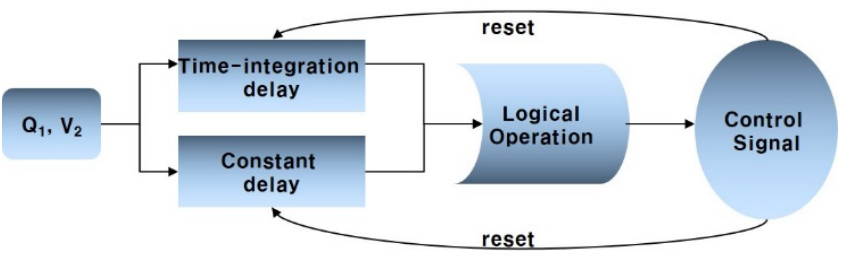

Fig. 6. The diagram of variable time delay control

First of all, the signal occurred quickly between them is selected. If the signal generated, the bus voltage is observed again within initializing the signal. The advantages of this method are that the rapid control is possible even if the voltage change is relatively large and near the deadband, thus the voltage profile is improved.

\section{Coordination Control between ULTC and S.C}

The operation objective of ULTC and S.C is the voltagereactive power control. Specifically, ULTC controls the voltage and S.C controls the reactive power. The voltage and the reactive power has an influence each other mutually, so that the operation of the device is thereby changing the operation conditions of other device. Overlapping of ULTC and S.C control can lead to voltage hunting at substation. This problem can frequently occur needless switching. Therefore, it requires the coordinated control of ULTC and S.C.

ULTC is used for the purpose of control the bus voltage of the secondary side by default, but S.C is based on the primary reactive power because the load-side receives the reactive power from network side. Since the amount is larger, the system is going to be vulnerable as the reactive power of network side is reduced. Therefore, it is suggested a control plane for coordinated control between ULTC and S.C, which is called as V2-Q1 plane in Fig. 7.

As a general rule, ULTC will change tap down or S.C will switch off on the high voltage. Coordinated control is necessary so as to prevent a confusion of ULTC and S.C cause system instability. An example of the voltage drop during a system disturbance is as shown in Fig. 8. In the coordination plane, ULTC control has wrong influence on

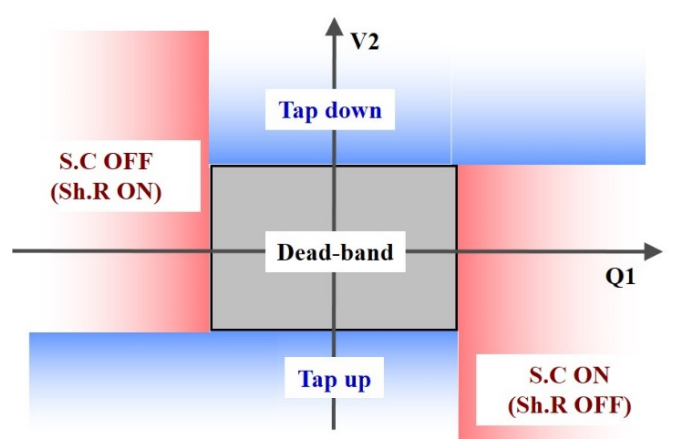

Fig. 7. V2-Q1 plane 


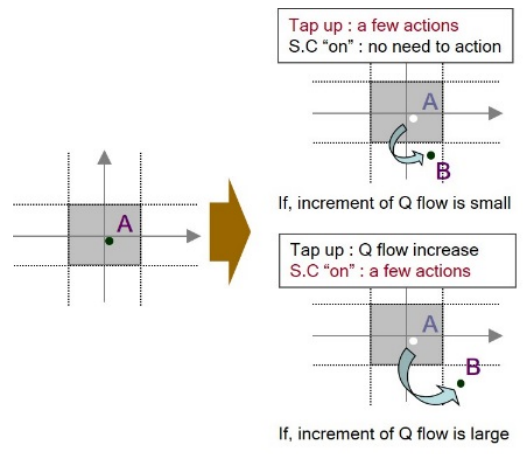

(a) Steady-State condition

(b) Fault occurs $(A \Rightarrow B)$

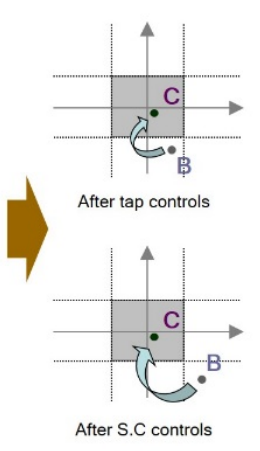

(c) Remedial actions $(\mathrm{B} \Rightarrow \mathrm{C})$
Fig. 8. Coordinated control of ULTC and S.C.

the system because the reactive power of the system goes to load. If Q1 is a bank of S.C, it switches on at outside of dead-band which is the point $\mathrm{C}$. And then the operating point goes to A or B, ULTC will change tap and control the voltage.

\section{Abnormal Condition Strategy}

ULTC control for maintaining the voltage from loadside aggravates the system condition when the system is near by the critical point. Therefore, ULTC and S.C/Sh.R control strategy should be established differently in the abnormal condition. System operator have to judge system condition which is stable or unstable in real-time, so it is needed the real-time VSI. If system will be unstable, the stability index exceeds the criterion. Conventional off-line voltage stability indices, for example, PV-curve and VQcurve, have limits for using on-line substation control. It is decided the voltage stability of the monitoring system by using the Z-index in real-time application. It requires information about voltage and current of only one bus that is a critical bus. The concept of the Z-index are as follows.

In order to observe the voltage stability of the power system, it should be noted the thevenin impedance changing in real-time first. The thevenin equivalent equation of Fig. 9 is as follows.

$$
\bar{E}_{t h}=\bar{V}+\bar{Z}_{t h} \bar{I}
$$

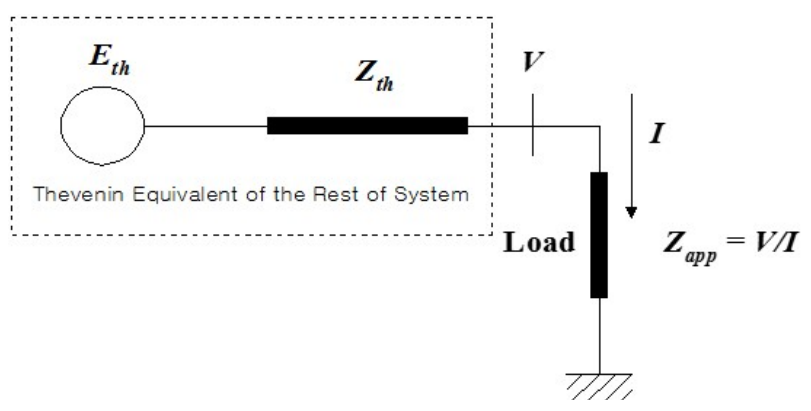

Fig. 9. The concept of the Z-index
The four variables of Eq. (2) are change as Eq. (3), and then it can be obtained from Eq. (2) to Eq. (4).

$$
\begin{gathered}
\bar{E}_{t h}=E_{r}+j E_{i} \\
\bar{Z}_{t h}=R_{t h}+j X_{t h} \\
\bar{V}=c+j d \\
\bar{I}=a+j b \\
{\left[\begin{array}{ccc}
1 & 0-a & b \\
0 & 1-b-a
\end{array}\right]\left[\begin{array}{c}
E_{r} \\
E_{i} \\
R_{t h} \\
X_{t h}
\end{array}\right]=\left[\begin{array}{l}
c \\
d
\end{array}\right]}
\end{gathered}
$$

It's impossible to solve the four variables of Eq. (4) because of lacking the number of equations even if the value of $a, b, c$, and $d$ are known. However, using that the circuit theory rule must be established at any point or time of operation, the solutions of it can be obtained by overlapping more than two equations with respect to the power system changing over time. By adjusting the size of the data windows, more than two equations are overlapped and the solution of Eq. (4) can be found when using a linear least square method. The thevenin impedance of the power system can be calculated through this process.

The power which the equivalent source can deliver to the load is maximum when the thevenin impedance is equal to the load impedance. Finally, the voltage stability of the power system is determined by the Z-index tracking in real-time which is in the ratio of the thevenin impedance and the load impedance. The $Z$-index has a value of 0.0 to 1.0. And the nearer the Z-index goes to the value of 1.0, the closer the local system will be unstable [14]. The condition of the Z-index are as follows.

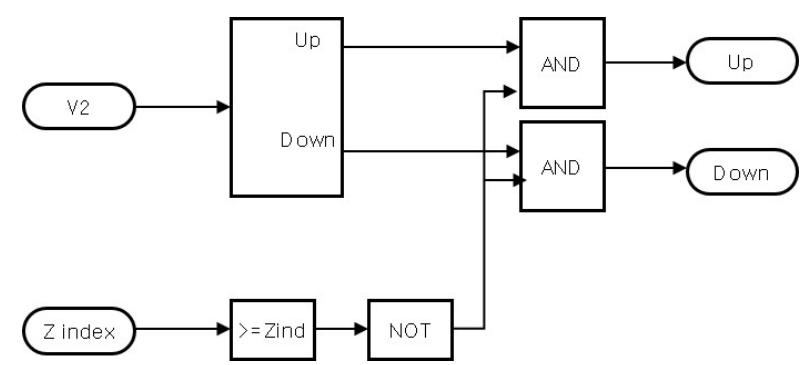

Fig. 10. The block diagram of the tap blocking

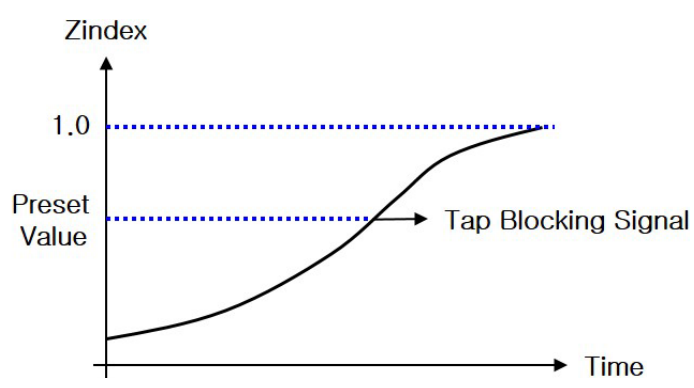

Fig. 11. The control scheme of the tap blocking 


$$
Z_{\text {index }}=\left|\frac{\bar{Z}_{\text {th }}}{\bar{Z}_{\text {app }}}\right| \quad\left\{\begin{array}{l}
\geq 1: \text { unstable } \\
<1: \text { stable }
\end{array}\right.
$$

If ULTC will react on an abnormal situation, it further grows voltage collapse. When the Z-index over the preset value, the tap block signal is sent to control center. The block diagram and the control scheme are as follows.

\section{Framework for the VRPC}

In this paper, we propose the efficient control strategy of ULTC and S.C/Sh.R at the local automated substation. Already mentioned it, the control strategy is the VRPC and the flowchart of the VRPC scheme is as follows.

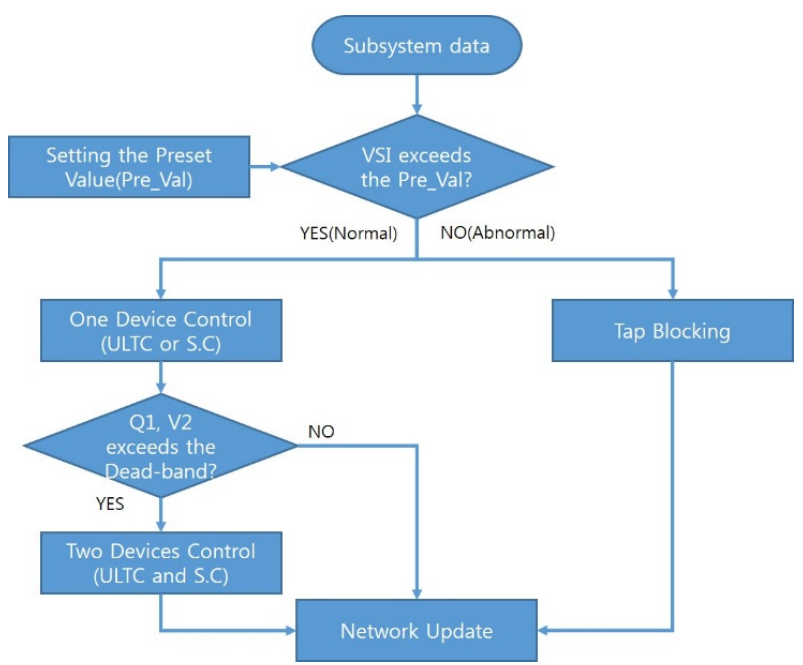

Fig. 12. The flowchart of the VRPC

First of all, set the preset value of the VSI and compare with the current VSI. If the current VSI exceeds the preset value, the system condition will go to the abnormal. So tap operation is blocking. When the current VSI don't exceed the preset value, just only one device is operated. At this time, if the monitoring value of the voltage or the reactive power over the dead-band, one device control is not need but coordinated control is needed.

\section{Case Study}

The proposed method is applied to BC Hydro-Quebec system. BC Hydro-Quebec system is made up the large amount of the load from south area and the generation part of the north area, so the active power is transmitted from the south area to the north area via interface lines. This situation is similar to the Korean power system. From this reason, BC Hydro-Quebec system is suitable for the simulations including the voltage instability and the voltage-reactive power control of the single substation when the load areas are observed [15]. The power-flow results of BC Hydro-Quebec system are as follows.

All of the hydro generators except MTL are located in the north area and two parts of the large load are situated in the south area. For verifying the useful of the VRPC, hierarchical substation construct at the single load that is

Table 1. The amount of the generation in BC HydroQuebec system

\begin{tabular}{c|c|c}
\hline Name & Active power [MW] & Reactive Power [MVAR] \\
\hline CHU & 5,000 & -26 \\
\hline LG2 & 4,936 & 335 \\
\hline LG3 & 1,800 & 240 \\
\hline LG3a & 180 & 24 \\
\hline LG4 & 2,500 & 141 \\
\hline MAN & 4,500 & -10 \\
\hline MTL & 4,000 & 409 \\
\hline Total & 22,916 & 1,112 \\
\hline
\end{tabular}

Table 2. The amount of the load in BC Hydro-Quebec system

\begin{tabular}{c|c|c}
\hline Name & Active power [MW] & Reactive Power [MVAR] \\
\hline MTL25 & 17,828 & $-1,057$ \\
\hline QUE25 & 3,944 & 2,026 \\
\hline Total & 21,772 & 969 \\
\hline
\end{tabular}

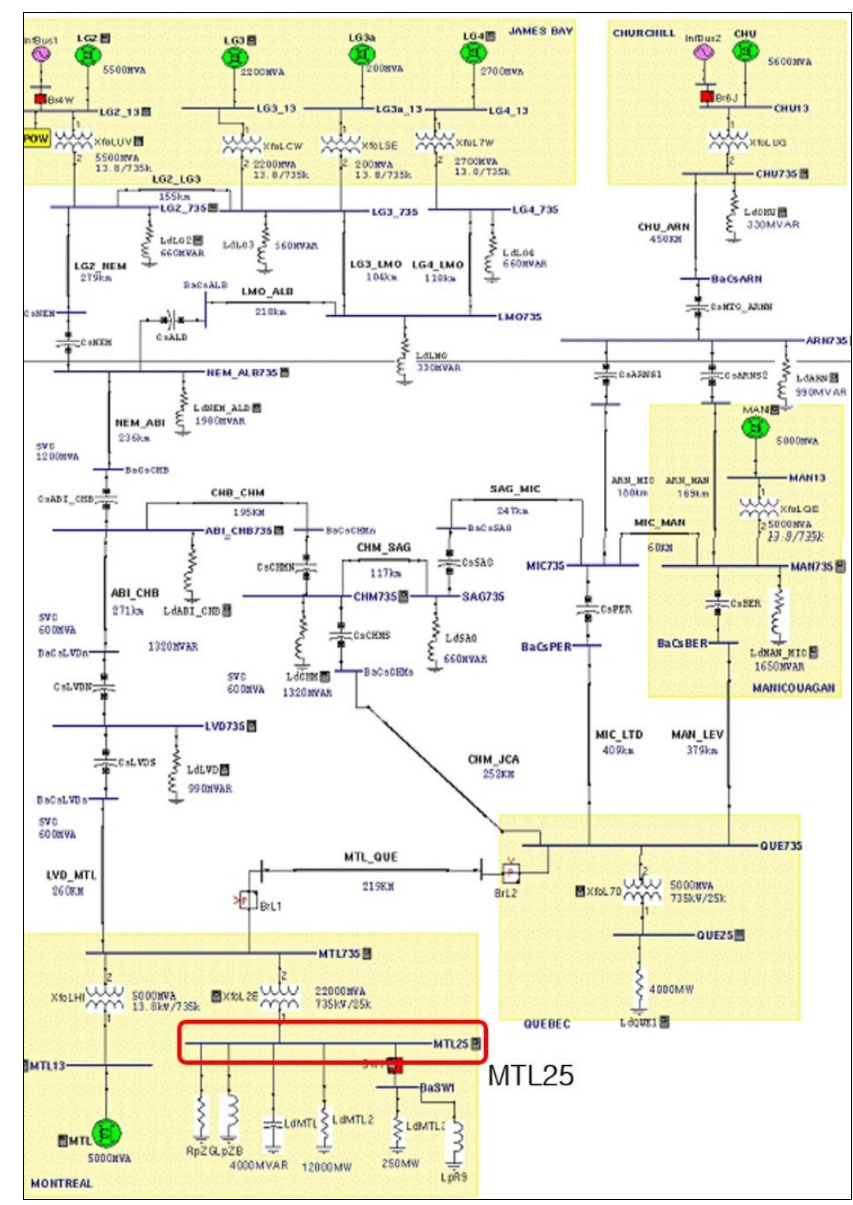

Fig. 13. BC Hydro-Quebec system 
MTL25 which is the monitoring and control bus. The controller of the VRPC acquires the current, the voltage, and the phasor information in real-time.

\subsection{Variable Time Delay}

In order to confirm effect of variable time delay method, load increase cases are applied to BC Hydro-Quebec system. The simulation scenarios and the results of the scenarios are as follows.

- After 2 seconds, load increase of 400MW and 400 MVAR at MTL25

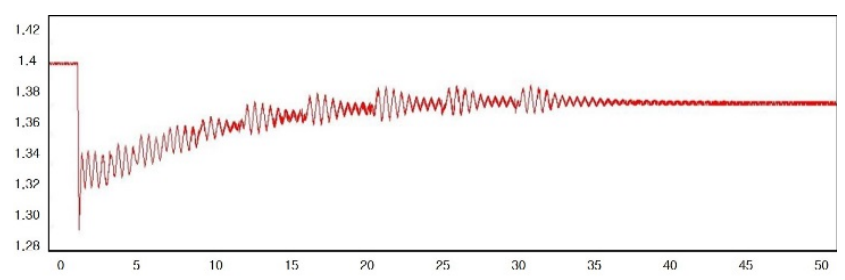

Fig. 14. Voltage magnitude of MTL25

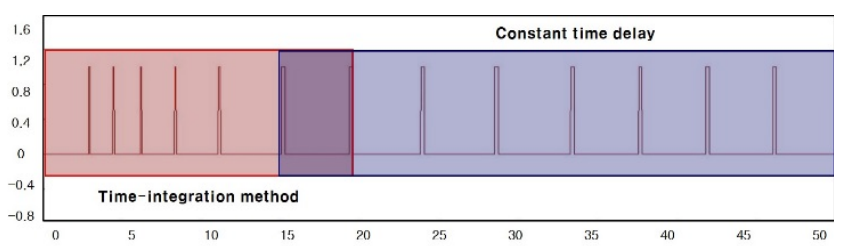

Fig. 15. Tap signal

The above are the results of ULTC actions. From 0 to $15 \mathrm{~s}$, the time integration method for large voltage error is used, since then uses constant time delay method for small voltage error. The actions of the constant time OLTC and the time integration OLTC seems to be in harmony.

\subsection{Coordination result}

In order to compare coordination control between ULTC and S.C, load increase cases are applied to BC HydroQuebec system. The simulation scenarios and the results of the scenarios are as follows.

- After 5 seconds, load increase of 400MW and 400 MVAR at MTL25

- After 15 seconds, load increase of 600MVAR at MTL25

After 5 seconds, tap signal occurs twice. Thus it can be seen that the bus voltage is controlled by ULTC. In 15 seconds, the reactive power increases as the load increase and become the outside of the Q1 dead-band. From this reason, ULTC is not operating owing to no tap signal. It can be seen that the voltage control by S.C in this area. It can be confirmed the coordination control between ULTC

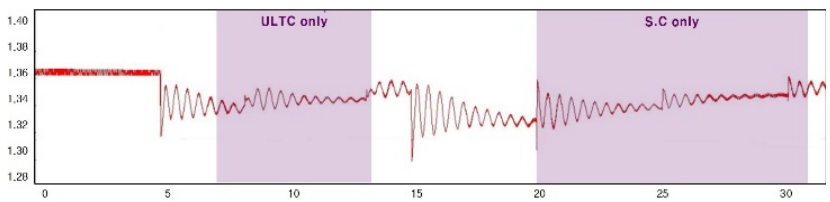

(a)

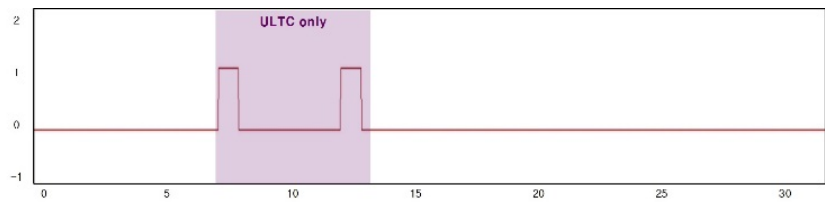

(b)

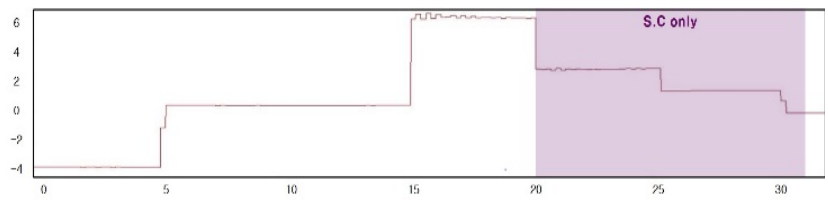

(c)

Fig. 16. (a) Voltage magnitude of MTL25; (b) Tap signal; (c) Reactive power flow

and S.C through the results.

\subsection{Abnormal condition result}

In order to compare effect of ULTC tap blocking for ongoing emergency situation, very severe cases are applied to BC Hydro-Quebec system. The simulation scenarios and the results of the scenarios are as follows.

- After 2 seconds, load increase of 400MW and 400 MVAR at MTL25

- After 10 seconds, line trip from MTL25 to QUE25

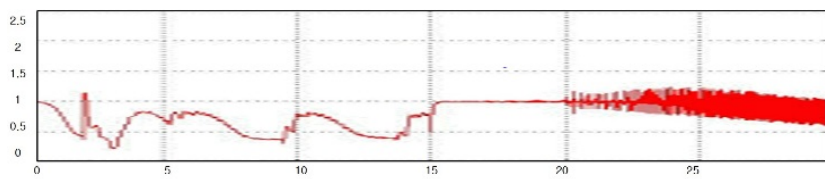

(a)

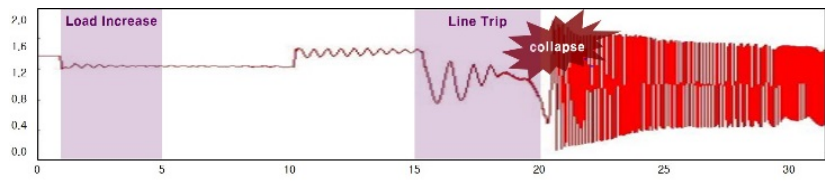

(b)

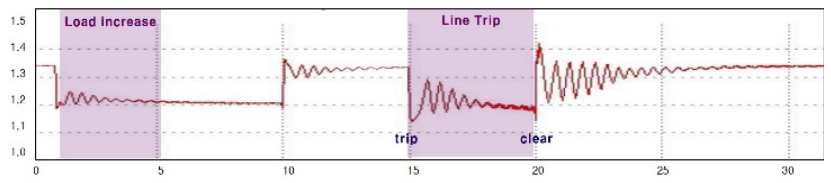

(c)

Fig. 17. (a) Z-index; (b) Tap acting at fault; (c) Tap blocking at fault 
It is known that the collapse point of the $\mathrm{Z}$-index value is 0.78 through off-line analysis. Since ULTC control continued when the Z-index is more than 0.78 , it can be seen that the system instability is accelerating. Although the low voltage occur, the system can be prevented collapse by tap blocking.

\section{Conclusion}

This paper proposed the automatic substation control method called the VRPC schemes for voltage and reactive power control. Through time-domain simulations for $\mathrm{BC}$ Hydro-Quebec system, the methodology will show the effectiveness in voltage-reactive power control and the accuracy in coordination of reactive power compensating devices including tap changer, shunt capacitor and shunt reactor.

For future works, an advanced control schemes and a more adequate index for Korean power system will be developed in the abnormal condition. Besides, there are more various coordinated controls, for example, FACTS device, load shedding, and etc.

\section{Acknowledgements}

This work was supported by the Brain Korea 21Plus Project in 2015 and the National Research Foundation of Korea (NRF) grant funded by the Korea government (MSIP) (No. 2011-0017650).

\section{References}

[1] C.W. Taylor, Power System Voltage Stability, McGrawHill Inc., 1994

[2] T. V. Cutsem, and C. Vournas, Voltage Stability of Electric Power Systems, Norwell: Kluwer, 1998.

[3] P. Kundur, Power System Stability and Control, McGraw-Hill, 1994

[4] D. Karlsson, and X. Waymel, "System protection schemes in power networks", Technical report, CIGRE Task Force 38.02.19

[5] M. Larsson, Coordinated Tap Changer Control, Theory and Practice, Lund 1997

[6] Z. Gajic, D. Karlsson, and M. Kockott, "Advanced OLTC Control to Counteract Power System Voltage Instability", ABB Power Technologies, Substation Automation, SE-721 59 VASTERAS, SWEDEN

[7] F. A. Viawan, A. Sannino, and J. Daalder, "Voltage control with on-load tap changers in medium voltage feeders in presence of distributed generation", Electric Power Systems Research, vol. 77, no. 10, pp. 1314-
1322, Aug. 2007

[8] C. Gao, and M.A. Redfern, "Automatic compensation voltage control strategy for on-load tap changer transformers with distributed generations", in Proceeding of International Conference on Advanced Power System Automation and Protection, October 16-20, 2011

[9] K.S. Jeong, H.C. Lee, Y.S. Baek, and J.H. Park, "Coordinated Voltage and Reactive Power Control Strategy with Distributed Generator for Improving the Operational Efficiency", Journal of Electrical Engineering \& Technology, vol. 8, no. 6, pp. 12611268, Nov. 2013

[10] Borka Milodevic, "On Voltage Stability and Control Using Multi-agent Systems", Ph.D. dissertation, Georgia Institute of Technology, November, 2002

[11] S. Seo, Y.H. Choi, S. Kang, B. Lee, J.H. Shin, and T.K. Kim, "Hybrid Control System for Managing Voltage and Reactive Power in the JEJU Power System," Journal of Electrical Engineering \& Technology, vol. 4, no. 4, pp. 429-437, 2009

[12] Y.H. Choi, B. Lee, S. Kang, and S.H. Kwon, "Multiple Substation Control using Voltage Stability Monitoring Index", in Proceeding of International Conference on Advanced Power System Automation and Protection, October 18-21, 2009

[13] I.C. Decker, M.N. Agostini, J.G. Ehrensperger, A.S. Silvia, A.L. Bettiol, and S.L. Zimath, "Synchronized Phasor Measurement System: Development and Applications", IX Symposium of Specialists in Electric Operational and Expansion Planning, May 23-27, 2004

[14] M. Begovic, B. Milosevic and D. Novosel, "A Novel Method for Voltage Instability Protection", in proceeding of the 35th Hawaii International Conference on System Sciences, Hawaii, USA, 2002

[15] Hypersim, Reference Guide Manual, TransEnergieTechnologies.

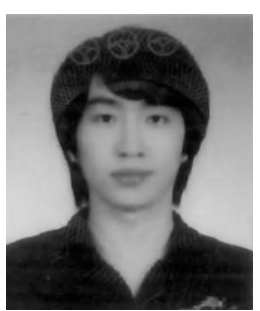

Yun-Hyuk Choi He received the B.S, M.S, and Ph.D. degrees in electrical engineering from Korea University, Seoul, Korea, in 2005, 2007, and 2012, respectively. From 2012 to 2014, he was a senior research engineer with the LSIS Corporation, Seoul, Korea. He is currently a research professor in the department of electrical engineering at Korea University. His main subjects are EMS application, distribution system engineering, and power system control. 


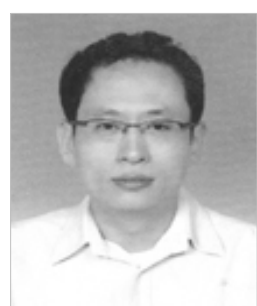

Sang-Gyun Kang He received the B.S, M.S, and Ph.D. degrees in electrical engineering from Korea University, Seoul, Korea, in 2003, 2005, and 2010, respectively. $\mathrm{He}$ is currently an assistant professor in the school of the electrical and railroad engineering at Kyungil University, Daegu, Korea. His interests are FACTS application, voltage stability analysis, and hardware power system modelling.

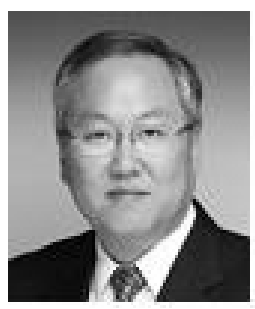

Byongjun Lee He received the B.S degree in electrical engineering from Korea University, Seoul, Korea, in 1987, and the M.S, and Ph.D. degrees in electrical engineering from Iowa State University, Ames, in 1991, and 1994, respectively. From 1995 to 1996 , he was a senior research engineer with the Mitsubishi Electric Corporation, Kobe, Japan. He is currently a professor in the department of electrical engineering at Korea University, Seoul, Korea. His responsibilities include power system operation, voltage stability analysis, system protection schemes, and wind farm application. 